\title{
COPeptin for diagnosis and prediction in Acute Coronary Syndrome (COPACS) Study: design and objectives
}

\author{
Beata Morawiec ${ }^{1}$, Damian Kawecki ${ }^{1}$, Lam Ho², Lui Chun Tat ${ }^{3}$, Olivier Muller ${ }^{4}$, Ewa Nowalany-Kozielska ${ }^{1}$ \\ ${ }^{1} 2^{\text {nd }}$ Department of Cardiology, School of Medicine with the Division of Dentistry in Zabrze, Medical University of Silesia in Katowice, \\ Poland \\ 2Department of Cardiology, Tuen Mun Hospital, Hong Kong, Hong Kong \\ ${ }^{3}$ Department of Accident and Emergency, Tuen Mun Hospital, Hong Kong, Hong Kong \\ ${ }^{4}$ Department of Cardiology, University Hospital, Lausanne, Switzerland
}

Adv Interv Cardiol 2016; 12, 4 (46): 360-363

DOI: 10.5114 /aic.2016.63627

\section{Introduction}

Chest pain is one of the most frequent and challenging clinical settings in emergency departments worldwide [1]. While the diagnosis and subsequent optimal management in ST segment elevation myocardial infarction (STEMI) are well established, rapid diagnosis of patients without persistent ST-segment elevation on ECG remains a challenge. Current guidelines on diagnosis and treatment of STEMI and non-ST-segment elevation acute coronary syndrome (non-STEACS) emphasize maximal shortening of time to diagnosis and treatment in high-risk patients [2-4]. Available tools are effective but time consuming. It is recommended to measure cardiac troponin, a gold standard marker of myocardial damage, in serial blood samples [3]. Copeptin, the C-terminal part of the prohormone for vasopressin, is emerging as a marker of acute endogenous stress. Due to its characteristic release pattern with an immediate increase a few minutes after symptom onset, together with cardiac troponin, a marker specific for myocardial injury, it could fill the gap and effectively shorten the time to diagnosis. Also in the context of early risk stratification crucial for optimal management, copeptin has high potential to be a rapid information tool $[5,6]$.

Below, we present the design of the COPACS (COPeptin for Acute Coronary Syndrome) Study, whose aim is to assess the role of copeptin in diagnosis and prediction of adverse events in a population of patients with chest pain without persistent T-segment elevation on ECG already at presentation, with regard to high-sensitive troponin T (hs-TnT).

\section{General information}

COPACS is a prospective observational study of the diagnostic and prognostic performance of copeptin in patients presenting to the Emergency Department with chest pain without persistent ST-segment elevation on ECG (non-conclusive ECG). Enrollment is conducted in the $2^{\text {nd }}$ Department of Cardiology, Zabrze, Medical University of Silesia, Katowice, Poland and is planned to be held in two rounds: 1) between December 15, 2011 and December 31, 2013, 2) between October 01, 2015 and December 31, 2016. Patients are enrolled according to prespecified inclusion and exclusion criteria (Table I).

Criteria of 6-hour chest pain was set as a result of previously published data on the profile of copeptin release in patients with acute coronary syndromes, which indicate rapid release with peak concentrations at $1-2 \mathrm{~h}$ from symptom onset followed by a decrease to the normal range up to 5-6 $\mathrm{h}$ [7]. This strategy follows guideline-recommended efforts for maximal shortening of the time from symptom onset to diagnosis and treatment in this group of patients.

Sample size. Based on retrospective analysis of patients admitted to the Emergency Department of the enrolling center in the 6-month period preceding the start of enrollment, the predicted number of included patients is 6-8 per month, which corresponds to a rate of 144-192 participants in the first round of enrollment and 234-312 participants at the end of recruitment.

\section{Study chart}

After inclusion, each patient undergoes initial clinical examination which includes physical examination,

\section{Corresponding author:}

Damian Kawecki PhD, $2^{\text {nd }}$ Department of Cardiology, School of Medicine with the Division of Dentistry, Medical University of Silesia, 10 Skłodowskiej-Curie St, 41-800 Katowice, Poland, phone: +48 32 2711010, e-mail: d.kawecki@interia.pl

Received: 29.09.2016, accepted: 17.10.2016. 
12-lead electrocardiogram (ECG), echocardiographic examination, and standard laboratory tests (blood count, sodium, potassium, creatinine, glomerular filtration rate (GFR), C-reactive protein, and N-terminal pro-B type natriuretic peptide (NT-proBNP)). High-sensitive troponin T, creatine kinase myocardial bound (CK-MB) and copeptin are measured at admission (TO). The initial diagnosis is established by the emergency physician and is verified by the supervisor cardiologist based on available data and ESC guidelines [3]. All patients are stratified according to the GRACE 1.0 risk score. Hs-TnT and CK-MB are measured afterwards at $6 \mathrm{~h}$ (T6) and repeated according to clinical indications. Furthermore, all included patients undergo routine diagnostic and therapeutic procedures as indicated in the ESC guidelines for non-STEACS [3]. Depending on the findings of myocardial necrosis markers and based on clinical judgment of the treating physician, the patient is qualified for a treadmill stress test or directly for a coronary angiogram (Figure 1 ).

\section{Measurement of copeptin}

Copeptin is measured from the blood sample drawn at admission (TO) and stored according to the instructions of the manufacturer. The result is double-blinded until completion of one-year follow-up. The measurement is performed using the BRAHMS Copeptin KRYPTOR assay on a BRAHMS KRYPTOR compact plus analyzer (BRAHMS $\mathrm{GmbH}$, Hennigsdorf, Germany) with the limit of detection 4.8-500 $\mathrm{pmol} / \mathrm{l}, 97.5^{\text {th }}$ percentile for healthy population $17.4 \mathrm{pmol} / \mathrm{l}, 20 \%$ coefficient of variation $12 \mathrm{pmol} / \mathrm{l}$. Copeptin is regarded as positive when $>17.4 \mathrm{pmol} / \mathrm{l}$, as recommended by the producer.

\section{Measurement of troponin}

Troponin used throughout the study is high-sensitive troponin T (hs-TnT). Measurement is carried out at admission (TO), after $6 \mathrm{~h}$ from admission (T6) and at further time points according to clinical indications and the deci-

Table I. Inclusion and exclusion criteria

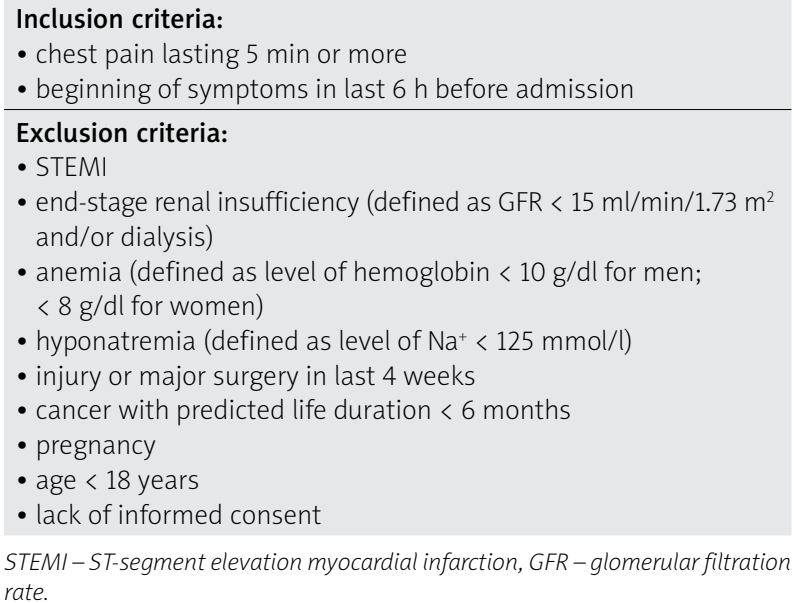

sion of the treating physician. High-sensitive troponin T is measured with electrochemiluminescent technology using the Elecsys Troponin T hs STAT assay (limit of detection 3-10 $000 \mathrm{ng} / \mathrm{l}, 99^{\text {th }}$ percentile for healthy population $14 \mathrm{ng} / \mathrm{l}(95 \% \mathrm{Cl}$ : 12.7-24.9 ng/l), 10\% coefficient of variation $13 \mathrm{ng} / \mathrm{l}$ ) on a cobase 411 analyzer (Roche Diagnostics GmbH, Mannheim, Germany). High-sensitive troponin $T$ is regarded as positive when $\geq 14 \mathrm{ng} / \mathrm{l}$, according to the manufacturer's indications and guidelines [3].

\section{Adjudicated final diagnosis}

Final diagnosis of non-ST-elevation myocardial infarction (NSTEMI), unstable angina (UA) or other causes of chest pain is established according to the independent opinions of two cardiologists, blinded to the copeptin result, after analysis of all available data and tests gathered during the hospital stay. In case of incoherence of their diagnosis, a third cardiologist is asked for an opinion.

\section{Follow-up}

Patients are monitored during a five-year period with clinical assessment conducted at discharge, 6 months, 1 year and 5 years. At 6-month and 5-year follow-up infor-

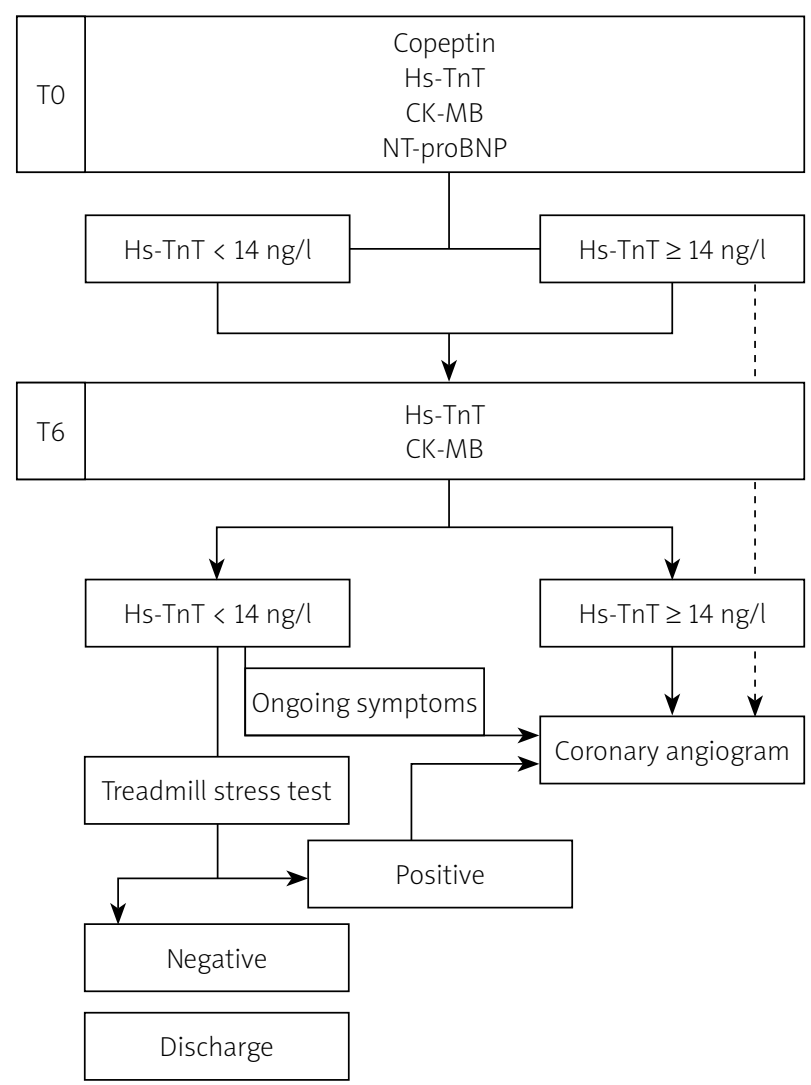

Figure 1. Study chart

TO - admission time, T6 - $6 \mathrm{~h}$ after admission, CK-MB - creatine kinase myocardial bound, NT-proBNP - N-terminal pro-B-type natriuretic peptide, $h s-\operatorname{Tn} T-h i g h$-sensitive troponin $T$. 
mation on Canadian Cardiovascular Society (CCS) class, New York Heart Association (NYHA) class and occurrence of study endpoints is collected in a phone call. One-year follow-up is conducted as a visit to the outpatient unit and involves physical examination, 12-lead ECG, echocardiogram, CCS and NYHA class determination, the blood test for NT-proBNP and information on the occurrence of study endpoints. If the phone contact fails, medical records of the enrolling center will be screened. If no information is found, national health care provider sources will be used to obtain information on hospitalization and endpoint occurrence.

\section{Endpoints}

All events are adjudicated by a cardiologist blinded to copeptin results. The primary diagnostic endpoint is the final diagnosis of NSTEMI.

The primary prognostic endpoint is defined as death of cardiovascular origin. The secondary prognostic endpoint is defined as major adverse cardiac and cerebrovascular events (MACCE) and includes death of cardiovascular origin, non-fatal acute myocardial infarction (AMI), $\mathrm{UA}$, repeated cardiac revascularization, and stroke.

Acute myocardial infarction is defined according to the third universal definition [8]. Repeated cardiac revascularization is defined as percutaneous coronary intervention or coronary artery bypass grafting on the vessel or lesion responsible for ACS at the moment of enrollment.

\section{Definitions}

Admission ECG is defined as non-conclusive if no persistent ST-segment elevation fulfilling the criteria of ST-segment elevation is present. ST-segment changes $(\Delta \mathrm{ST})$ are defined as depression of the ST segment of $\geq 1 \mathrm{~mm}$ or non-persistent ( $<20 \mathrm{~min}$ ) elevation of the ST segment of $\leq 1 \mathrm{~mm}$ or $\mathrm{T}$ wave reversal $>2 \mathrm{~mm}$, each of these in at least two contiguous leads in 12-lead ECG. Coronary artery disease is diagnosed in the coronary angiogram if the stenosis of the coronary artery is $>50 \%$. Maximal hs-TnT and maximal CK-MB are defined as the maximal concentrations of the biomarkers measured during the hospital stay.

\section{Statistics}

Continuous variables are presented as mean \pm standard deviation or median (interquartile range) and are compared with Student's $t$ test or the Mann-Whitney $U$ test and ANOVA or Kruskal-Wallis test, depending on their distribution. Categorical variables are presented as $n(\%)$ and are compared with the $\chi^{2}$ test. Spearman and Pearson methods are used for calculating the correlation of copeptin with continuous and categorical variables, respectively. Follow-up is planned to be continued until oc- currence of an endpoint or up to 5 years from enrollment. Survival curves and MACCE-free curves for copeptin, hs-TnT and their combinations are performed with the Kaplan-Meier method. Diagnostic accuracy of copeptin, hs-TnT and their combination for prediction of endpoints is presented as receiver operating characteristic (ROC) curves. Areas under the curve (AUC) are compared with the $z$ test. The influence of biomarkers and preselected baseline clinical and procedural parameters on the occurrence of endpoints is calculated with uni- and multivariable Cox proportional hazard regression models.

A novel statistical method - taxonomy - will be used to evaluate the predictive accuracy of copeptin. The additional value of taxonomy over commonly used regression models is the ability to identify a subgroup of patients with the highest risk in relation to the studied variable (here copeptin) regarding the defined study endpoint. As previously described [9], taxonomy is a form of multivariate analysis based on correlation of prespecified variables. As a result it provides clusters, groups of patients with minimal intragroup similarities and maximal intergroup differences in parameters, defined by "distance". According to Marczewski and Steinhaus [10], the distance equals the symmetric difference between patients in studied variables. With defined distance between variables, linkage methods are used to link sufficiently similar clusters together, to finally form a classification tree, a dendrogram. This graphic presentation of multidimensional space of patients and variables is regarded to be useful in identification of factors predictive for variables differentiated in this model. Intercluster differences in studied parameters are analyzed with one-tailed variance, and the Mann-Whitney $U$ test is used for analogous analysis of the endpoint.

The $p$-value of $<0.05$ will be assumed significant throughout the analysis. All analyses are performed with Statistica software 10 (StatSoft Inc., Tulsa, OK, USA), GraphPad Prism 6.00 (GraphPad, La Jolla, California, USA) and platform R, v. 3.0.2 (R Foundation for Statistical Computing, Vienna, Austria).

\section{Ethics}

The study received approval from the Ethical Committee of the Medical University of Silesia (decision no. KNW/0022/KB1/187/11) and is conducted according to the policy of the Declaration of Helsinki. All patients who met the inclusion criteria gave their informed consent before inclusion in the study.

\section{Study registration number \\ ISRCTN14112941 (www.isrctn.com).}

\section{Conflict of interest}

The authors declare no conflict of interest. 


\section{References}

1. Mockel M, Searle J, Muller R, et al. Chief complaints in medical emergencies: do they relate to underlying disease and outcome? The Charite Emergency Medicine Study (CHARITEM). Eur J Emerg Med 2013; 20: 103-8.

2. Steg G, James SK, Atar D, et al. The Task Force on the management of ST-segment elevation acute myocardial infarction of the European Society of Cardiology (ESC). ESC Guidelines for the management of acute myocardial infarction in patients presenting with ST-segment elevation. Eur Heart J 2012; 33: 2569-619.

3. Hamm CW, Bassand JP, Agewall S, et al. The Task Force for the management of acute coronary syndromes (ACS) in patients presenting without persistent ST-segment elevation of the European Society of Cardiology (ESC). ESC Guidelines for the management of acute coronary syndromes in patients presenting without persistent ST-segment elevation. Eur Heart J 2011; 32: 2999-3054.

4. The Task Force on Myocardial Revascularization of the European Society of Cardiology (ESC) and the European Association for Cardio-Thoracic Surgery (EACTS). Guidelines on myocardial revascularization. Eur Heart J 2010; 31: 2501-55.

5. Khan SQ, Dhillon OS, O'Brien RJ, et al. C-terminal provasopressin (copeptin) as a novel and prognostic marker in acute myocardial infarction: Leicester Acute Myocardial Infarction Peptide (LAMP) study. Circulation 2007; 115: 2103-10.

6. Voors AA, von Haehling S, Anker SD, et al. OPTIMAAL Investigators. C-terminal provasopressin (copeptin) is a strong prognostic marker in patients with heart failure after an acute myocardial infarction: results from the OPTIMAAL study. Eur Heart J 2009; 30: 1187-94.

7. Gu YL, Voors AA, Zijlstra F, et al. Comparison of the temporal release pattern of copeptin with conventional biomarkers in acute myocardial infarction. Clin Res Cardiol 2011; 100: 1069-76.

8. Thygesen K, Alpert JS, Jaffe AS, et al.; Joint ESC/ACCF/AHA/WHF Task Force for Universal Definition of Myocardial Infarction. Third universal definition of myocardial infarction. J Am Coll Cardiol 2012; 60: 1581-98.

9. Tukiendorf A, Kaźmierski R, Michalak S. The taxonomy statistic uncovers novel clinical patternsin a population of ischemic stroke patients. PLoS One 2013; 8: e69816.

10. Marczewski E, Steinhaus $H$. On a certain distance of sets and the corresponding distance of functions. Colloq Math 1958; 6: $319-27$. 